\title{
Our experience in intratympanic vs intravenous steroids for Idiopathic Sudden Sensorineural Hearing Loss
}

\author{
S. Y. Kosyakov*, A. V. Gunenkov*, A. G. Atanesyan*, E. R. Ashkhatunyan**, \\ * Russian Medical Academy of Postgraduate Education, ENT chair \\ 7 Ivan'kovskoe shosse, Moscow, Russia \\ ** Ministry of Healthcare and Social Development of the Russian Federation
}

\section{No grant support.}

\section{Abbreviations:}

Dex - dexamethasone

ISSNHL - idiopathic sudden sensorineural hearing loss

IT - intratympanic

IT-Dex - intratympanic dexamethasone

IV-Dex - intravenous dexamethasone

PTA - pure-tone audiometry

ST - standard reologic and steroid therapy
$\mathrm{PTA}_{\mathrm{ALL}}$ - pure tone average on all frequencies $\mathrm{PTA}_{\mathrm{LF}}$ - pure tone average on low frequencies $\mathrm{PTA}_{\mathrm{MF}}$ - pure tone average on medium frequencies $\mathrm{PTA}_{\mathrm{HF}}$ - pure tone average on high frequencies.

Keywords: idiopathic sudden sensorineural hearing loss, sudden sensorineural hearing loss, sudden deafness, intratympanic steroids.

\begin{abstract}
Background. Currently the treatment idiopathic sudden sensorineural hearing loss by steroids is considered to be the most effective and common one. It is established that intratympanic administration of steroids, even in small quantities, results in its higher concentration in the end organ compared with systemic administration. Therefore drugs that are used topically in low doses are to be preferred. We will discuss our experience for intratympanic treatment as opposed to systemic steroids

Methods. 73 patients were observed in the study. 24 patients among them were treated with intratympanic dexamethasone over a period of 6 months. 24 patients were treated with steroids and reologic substances and 25 patients were treated with intravenous dexamethasone only over a period of 10 days. Pretreatment and 1-, 3-, 6-month post-treatment using pure-tone audiograms were compared.

Results. Intratympanic and systemic therapy did not show any differences in the treatment efficiency during the first month. But intratympanic steroid therapy resulted in higher efficacy after prolonged treatment, i.e. 6 months. No differences were found between groups treated with systemic steroid monotherapy or with steroids and reologic substanses.

Conclusions. Long-term IT steroid therapy over a period of 6 months showed a more noticeable efficacy than with steroids and reologic substanses and systemic steroid monotherapy. Our experience confirms that IT therapy can be used as the primary method for treatment for patients with contraindications of systemic steroid therapy and for those with mainly high-frequency sensorineural hearing loss.
\end{abstract}

\section{Introduction}

Though there is no universal definition, ISSNHL is often defined as unexplained sensorineural hearing loss $30 \mathrm{~dB}$ or greater in 3 or more contiguous audiometric frequencies occuring within 72 hours. Sometimes it is clarified as unilateral and without pronounced vertigo. Steroid therapy is considered to be the most effective and common method for ISSNHL treatment alone or combined. This is confirmed in national guidelines, e.g. in Germany, Russia, Spain and the USA.

However the treatment remains challenging. Neither the optimal dosage of systemic steroids nor the treatment duration has been precisely defined, so they are often chosen empirically [1]. Paper [2] clarifies this issue: for maximum treatment outcomes the recommended doses of oral prednisone are given as $1 \mathrm{mg} / \mathrm{kg} / \mathrm{d}$ in a single dose, with the usual maximum dose of $60 \mathrm{mg}$ daily, with a treatment duration of 10 to 14 days. Data comparing treatment protocols are limited, but one representa- 
tive regimen uses the maximum dose for 4 days, followed by a 10-mg tapering off every 2 days [3]. This dose is based on a maximum adrenal output of hydrocortisone (cortisol) of 200 to $300 \mathrm{mg} / \mathrm{d}$ during stress. Prednisone is 4 times, methylprednisolone is 5 times, and dexamethasone is 25 times more powerful than hydrocortisone. So the equivalent dose is for prednisone is $60 \mathrm{mg}$, $48 \mathrm{mg}$ for methylprednisolone and $10 \mathrm{mg}$ for dexamethasone [1].

Common side effects of glucocorticoids include insomnia, dizziness, weight gain, increased sweating, gastritis, mood changes, photosensitivity, and hyperglycemia. Severe (but rare) side effects include pancreatitis, bleeding, hypertension, cataracts, myopathy, opportunistic infections, osteoporosis, and osteonecrosis manifesting as fractures and aseptic necrosis of the femoral and humeral heads [1]. This means patients with systemic medical conditions such as insulin-dependency or poorly controlled diabetes, labile hypertension, tuberculosis, peptic ulcer disease, and prior psychiatric reactions to corticosteroids, among others, may not be suitable to receive systemic corticosteroids.

Another issue far from being evident is IT application of corticosteroids. On one hand the benefit of this route seems now to be obvious. Parnes et al. [4] published animal studies and clinical series and demonstrated higher inner ear steroid levels following IT steroid application, with benefit in one-third of patients, and higher percentages of benefit in certain otologic conditions. Subsequent laboratory data has substantiated the claim of higher perilymph steroid concentrations after IT steroid application [5].

However prospective, randomized papers showed that intratympanic treatment with 4 doses over 14 days of $40 \mathrm{mg} / \mathrm{mL}$ of methylprednisolone injected into the middle ear was no less effective than the oral prednisone treatment [6].

The IT application of steroids, even in low dosage, leads to a higher drug concentration in the inner ear fluids when compared with systemic administration [5-11].

The IT administration of steroids is associated with a low rate of side effects These are infrequent but include pain, transient dizziness, infection, persistent tympanic membrane perforation, possible vasovagal or syncopal episodes during injection, and in comparison with the oral route, higher cost, and multiple office visits $[1,12,13]$.
IT application is approved in the national guidelines mentioned above.

The objective of our study is to evaluate the effectiveness of the IT application of steroid treatment in patients with ISSNHL compared with the IV application of steroids and ST.

The permission of ethical committee was obtained for our clinical research.

\section{Materials and Methods}

Only patients with ISSNHL were included in the study. The analysis of the therapy efficacy was performed on 73 patients (mean age $43.4 \pm 11.9 \mathrm{SD}$ yr; range, 23-69 yr), consisting of 31 women and 42 men, all without previous treatment. Two of them demonstrated bilateral impairment.

The inclusion criteria during this study was as follows: patients with ISSNHL, who had not previously been treated and were older than 18 years. The time between the onset and the beginning of the therapy was less than 1 month.

The following exclusion criteria were adopted:

1. Patients with somatic pathology (such as diabetes, hypertension, gastriculcer, tuberculosis, glaucoma, and so on), for whom systemic steroids were contra-indicated;

2. oncological patients;

3. patients with autoimmune diseases or those who were constantly or periodically taking steroids;

4. pregnant and nursing women;

5. patients with middle ear diseases, abnormal type of tympanometric curves or barotrauma in their medical history;

6. those who had intolerance for any component of treatment;

7. those who had ISSNHL in the only hearing ear. All patients were divided into 3 groups depending on the method of therapy. The division into groups was based on mechanical randomization.

The IT-Dex group of 24 patients (25 ears) were treated with Dex intratympanically. Dex was injected through a tympanostomy tube fixed in the posteroinferior quadrant of the tympanic membrane under the local anesthesia. Dex was given according to the following dosage regimen: $4 \mathrm{mg}$ (one ampoule of $1 \mathrm{ml}$ ) every day during 10 days, $4 \mathrm{mg}$ every other day over 20 days and then $4 \mathrm{mg} 2$ times a week over 5 months. Injecting the drug in the tympanic cavity through the tympanostomy tube, we oriented patient's head in a specific position to be sure the round window membrane was covered 
with the solution for 30 minutes. We asked the patient to avoid swallowing to reduce the incidence of the drug leaking through the eustachian tube.

The ST group consisted of 24 patients (25 ears) who received for 10 days Pentoxifylline, Cocarboxylase, Potassium and magnesium aspartate intravenously and vitamin B1/B6/B12-complexe intramuscularly and Dex $0.1 \mathrm{mg}$ for $1 \mathrm{~kg}$ of body weight in $200 \mathrm{ml}$ of $0.9 \%$ sodium chloride solution intravenously tapering off over 5 days.

The IV-Dex group consisted of 25 patients (25 ears) who were treated with Dex $0.1 \mathrm{mg}$ for $1 \mathrm{~kg}$ of body weight in $200 \mathrm{ml}$ of $0.9 \%$ sodium chloride solution intravenously daily over 5 days tapering off over the following 5 days .

The evaluation of the therapy efficacy was based on the PTA data before and after treatment. Hearing evaluation was based on the following criteria: A hearing restoration within $15 \mathrm{~dB}$ was defined as a complete recovery. An improvement of the average hearing of $50 \%$ or more from the initial test results as a partial recovery and a reduction of hearing thresholds of $15 \mathrm{~dB}$ or more as a hearing improvement. The average hearing levels were determined by 4 frequencies $(500,1000,2000$ and $4000 \mathrm{~Hz}$ ).

All patients had a follow-up period of 6 months. They were observed before the treatment, 10 days after, and then 1, 3 and 6 months after the start of the therapy.

Statistical data processing was performed with the software Statistica (StatSoft Inc., release 6.1) and Biostatistics, Version 4.03, by Stanton A. Glantz, USA 1998. The statistical analysis included the following methods: descriptive statistics, analysis of variance (One-Way ANOVA), repeated measures analysis of variance, Newman-Keuls test, pared t-test, Kruskal-Wallis test, Dunn test, ChiSquared test, Fisher exact test (one-tailed version), factor analysis of variance, analysis of covariance (ANCOVA), logistic regression, polynomial regression.

\section{Results}

There were no significant differences in sex, age, PTA (including low-, mid- and high-frequency ranges), duration between onset and treatment of the disease, association with vertigo or hearing loss degree between the three groups $(p<0.05)$.

The therapy efficacy was evaluated 1 and 6 months after the treatment beginning. At the first stage of our study, the results were divided into 2 subgroups depending on presence or absence of treatment response: with positive effect («effect+» subgroup) and without positive effect («effect-» subgroup). «Effect+» subgroup included cases of complete recovery, partial recovery and hearing improvement, whereas «effect-» subgroup consisted of cases without changes or with hearing loss.

After one month the treatment started no significant differences between groups was discovered. However 6 months after the beginning of the therapy we found a significant difference $\left(\chi^{2}, p<0.05\right)$. The IT-Dex group represented the major component of positive clinical results $(88 \%)$, whereas the ST and the IV-Dex groups differed slightly ( $48 \%$ and $56 \%$ respectively), which was considered to be insignificant. The difference between the IT-Dex group and the two others overall was significant $\left(\chi^{2}, p<0.05\right)$. At the second stage the analysis of previously revealed differences and further evaluation of the significance of differences between groups was performed. The comparative analysis of rates of complete recoveries, partial recoveries, hearing improvements («effect+») subgroup) and of cases without changes or with hearing loss («effect-» subgroup) in all groups 6 months after the therapy beginning revealed the greatest differences within the complete recovery rates between groups - in the IT-Dex group, $60 \%$ of patients demonstrated complete recovery, while in the StT group, as well as the IV-Dex group only $20 \%$ of patients recovered completely.

6 months after the treatment beginning the following results were obtained:

1. Differences of complete recoveries rates between IT-Dex group and ST group were significant $(\chi 2, \mathrm{p}<0.05)$.

2. Differences of complete recoveries rates between IT-Dex group and IV-Dex group were significant $(\chi 2, p<0.05)$.

3. No significant differences of complete recoveries rates were seen between ST and IVDex groups.

Thus, in 6 months the highest efficacy was observed in the group of patients treated with IT steroids (the maximal rate of complete and partial recoveries and of hearing improvements) compared to the group of patients who had received ST therapy and systemic steroid monotherapy. The number of patients with complete recovery in the IT group was significantly higher than in the two other groups 6 months after the treatment beginning. 
Moreover, the effect of the therapy on different frequencies (low, middle and high) was evaluated. Frequency range of 125 and $250 \mathrm{~Hz}$ was defined as low frequencies; 500, 1000, $2000 \mathrm{~Hz}$ - as medium frequencies and $4000,8000 \mathrm{~Hz}$ - as high frequencies. Pure tone thresholds in average on these bands $\mathrm{PTA}_{\mathrm{LF}}, \mathrm{PTA}_{\mathrm{MF}}, \mathrm{PTA}_{\mathrm{HF}}$ and on all the frequencies PTA $_{\text {ALL }}$ were studied.

The PTA $_{\mathrm{ALL}}$ decrease in the different phases of treatment is presented in fig.1. In the IT-Dex group the maximal effect was observed 10 days after the beginning of the treatment. The PTA decrease 10 days after the therapy beginning and later (1, 3 and 6 months after), in comparison with the initial hearing level was significant $(\mathrm{p}<0.05)$. The PTA ${ }_{\mathrm{ALL}}$ decrease 6 months after the treatment beginning compared with one month after the treat- ment beginning was also significant $(\mathrm{p}<0.05)$. The maximal effect in the ST group was also observed 10 days after the beginning of the treatment. The following changes of PTA $_{\mathrm{ALL}}$ during the period from the 10th day to the 6th month of therapy were not significant. In the IV-Dex group, as well as in the ST group, the maximal effect was observed 10 days after the beginning of the treatment and the following changes of PTA $_{\mathrm{ALL}}$ during the period from the 10th day to the 6th month of therapy were also not significant.

Thus, in the IT-Dex group improvement was registered during the whole period of the 6 months treatment, whereas in the two other groups patients had an improvement only during the first 10 days of the therapy (fig. 1).

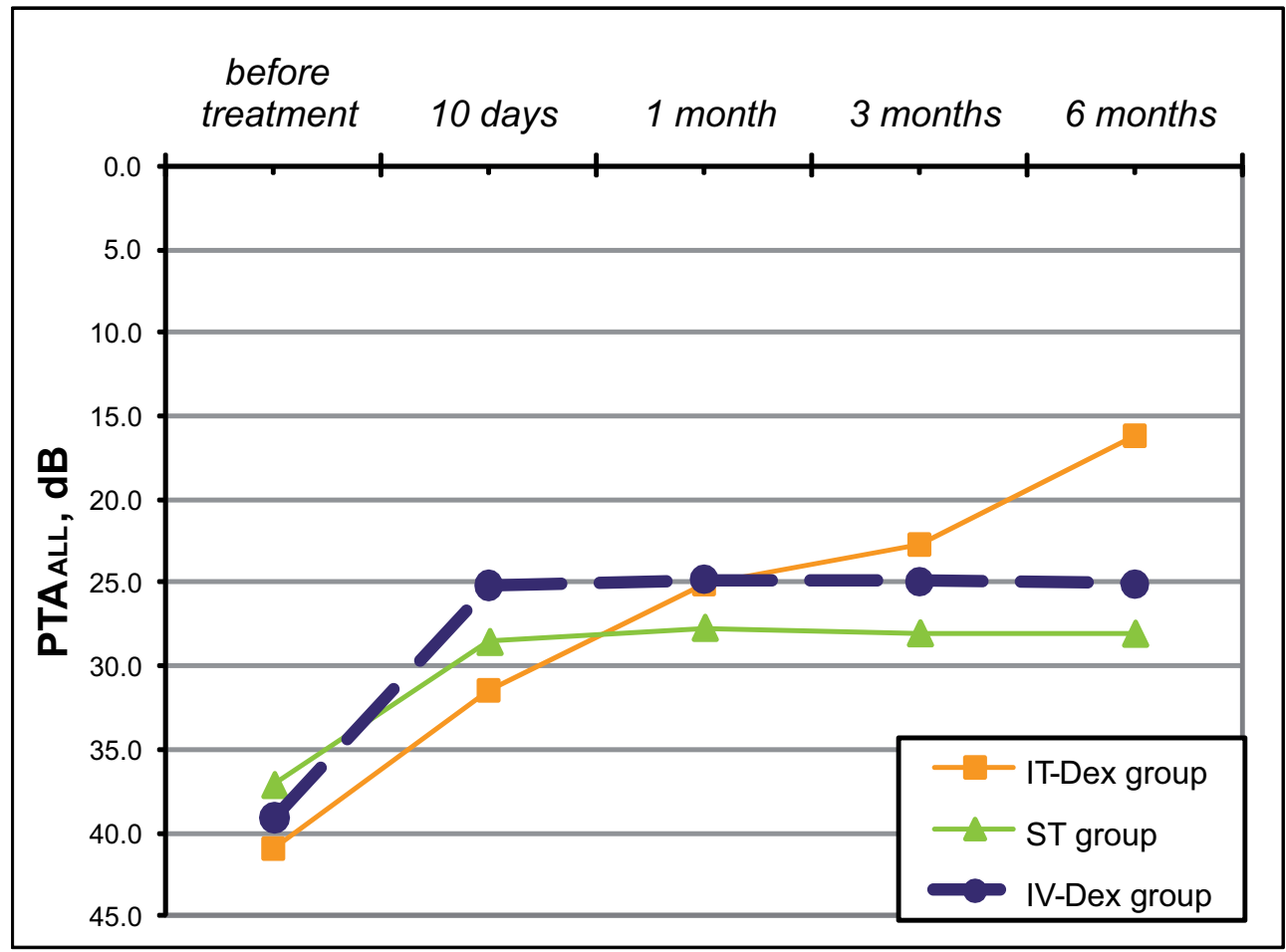

Fig. 1. Hearing changes in 3 groups during 6 months.

The analysis of variance was used to evaluate the significance of the differences of therapy efficacy within different frequency ranges in three groups 1 and 6 months after the treatment's beginning. It was found that 1 month after the therapy beginning there were significant differences between groups over the high-frequency range, whereas 6 months after - on all frequencies $(\mathrm{p}<0.05)$. Further analysis demonstrated that this difference over the high-frequency range 1 month after the treatment's beginning was due to a more expressed effect ( $p$ $<0.05)$ in the IT-Dex group compared to the ST group. Differences between the ST and IV-Dex groups were not significant.

At the same time, 6 months after the treatment's beginning the PTA changes in the IT-Dex group were more noticeable than in the ST and IV-Dex groups within the high-frequency range and on all 
frequencies overall $(\mathrm{p}<0.05)$. No significant differences in PTA changes on all frequencies separately and in total between ST and IV-Dex groups were found $(\mathrm{p}>0.05)$.

Thus, after 1 month of therapy, differences between groups were valid only for high frequencies because of a significantly greater efficacy in the IT-Dex group compared to the ST group. Six months after the IT-Dex group demonstrated a better effectiveness over the high-frequency range and on all frequencies in total compared to the two other groups. IV-Dex and ST didn't differ from each other for all mentioned above criteria.

Evaluation of factors associated with therapy efficacy.

The association of the treatment efficacy with patient's age, degree of hearing loss, start up time of therapy and vertigo was evaluated.

Vertigo and hearing loss degree were not associated with the therapy efficacy. Patient's age and the time between the onset of ISSHNL and the beginning of the therapy seemed to influence the effectiveness of the treatment. The rate of complete recovery was lower in patients above 35 years old and with a timescale between the onset and the beginning of the treatment of more than 7 days.

\section{Discussion}

Our study demonstrated an identical treatment efficacy of the IT and IV administration of steroids and the ST 1 month after the treatment commenced. Despite the absence of differences in one-month therapy efficacy in general between groups, the analysis of the impact on different frequencies revealed a better effectiveness of IT administered steroid on high frequencies compared with standard therapy. A. N. Salt and S. K. Plontke postulated that agents, delivered to the round window membrane locally, were not regularly distributed in the inner ear, but demonstrated a base-to-apex gradient of concentration, so that basal parts of the cochlea received far greater medicine concentrations than apical parts $[8,11]$. This fact seems to explain the greater efficacy of IT steroids over the high-frequency range.

Patients who were treated with IT steroids demonstrated an improvement (PTA decrease on all frequencies) during the whole treatment period, contrary to those from the 2 other groups treated systemically, who improved only during the first 10 days of the therapy. This data lets us suppose a potential possibility of hearing improvement resulting from a longer course of treatment. The duration of treatment with systemic steroids is limited because of their side effects.

Many studies indicate that spontaneous recovery occurs in $30-65 \%$ of cases [1, 16-21]. Generally, the recovery happens within 2 weeks after the disease onset [17]. H. Zhao et al. claim that the treatment which was started within 2 weeks after the onset is more effective than the one after a fortnight of the onset and later [22].

According to our data the absence of positive response after 10 days of therapy (1, 3, 6 days after) in the groups of patients treated with systemic steroids over 10 days argues for the poor prognosis of recovery without treatment at a later date. Due to the significant differences of $\mathrm{PTA}_{\mathrm{ALL}}$ obtained in our study in test time, long-term IT administration of steroids has a higher justification.

In the IT-Dex group the efficacy of the treatment was more significant than in the ST and IV-Dex groups 6 months after the beginning of the therapy. Furthermore, the rate of complete recoveries 6 months after the treatment beginning was higher for local therapy than for standard and systemic steroid therapy. This is also a proof of the capacity of hearing restoration after a longer course of treatment.

The chance of hearing improvement at the later times of therapy has been demonstrated by many authors, pointing the effectiveness of IT administration of steroids after an earlier inefficient systemic therapy [22-28].

Our results of equal efficiency of therapy for all the 3 protocols 1 month after the treatment commencement allows us to confirm the IT administration of steroids as an independent treatment mode. Generally, it concerns patients having contraindications against the systemic steroid therapy. Similar data were received by Y. Peng et al. They found that patients with hearing loss less than $70 \mathrm{~dB}$ showed no difference between the effectiveness of local and systemic steroid application, whereas those patients who had hearing loss more than $70 \mathrm{~dB}$ showed a better response to the local therapy [27]. In our study only one patient from IT-Dex group had a hearing loss more than $70 \mathrm{~dB}$. However S. Kakehata et al. obtained a higher efficacy of local steroid treatment over 8 days compared to systemic steroid therapy in patients with diabetes [28].

In contradiction with the majority of aforementioned guidelines we prefer intravenous perfusion 
but not per os for the systemic therapy. That was due to national custom that is confirmed in national guidelines for ISSNHL treatment and we didn't find studies for the superiority of one over another. No serious side effects related to systemic administration of steroids were observed in our study. 9 patients in ST group and 12 patients in IV-Dex group complained of sleep loss, which was completely corrected after withdrawal. That confirms the opinion that most serious side effects occur with chronic use, and adverse events are usually acceptable and manageable for the short 10- to 14-day course of steroids recommended for ISSNHL. Alexander et al. [29] reviewed the safety of high-dose steroids taken for up to 22 weeks for autoimmune inner ear disease and found that most patients completed the course, with the most frequent adverse events being hyperglycemia and weight gain. There is also evidence that osteonecrosis and fractures occur more commonly in patients with preexisting bone or joint problems in conditions such as systemic lupus erythematosis and rheumatoid arthritis [30].

Optimal dosage of steroids for IT administration has never been precisely estimated and treatment protocols for local steroid application have not yet been designed. Also we couldn't set an optimal duration of local treatment in our study, because the obtained data (improvement during all the period of treatment -6 months, and different number of patients with complete hearing recovery at different moments of treatment) show that this duration is variable and specific to each patient. Therefore, it might be reasonable to continue the treatment as long as an improvement is observed, for example, until obtaining 2 similar audiograms spaced a month apart with the hearing survey being done monthly. However in these cases the outcome (benefit and harm) could be partially due to swallowing of steroids via eustachian tube during therapy, meaning this is not local in fact. In this link there are papers generating some controversy about systemic effects of IT glucocorticoids [31]. In animal studies, ITDex infusions resulted in higher perilymph concentrations than intravenous dexamethasone application without any systemic absorption [32]. As far as humans are concerned, small concentrations of methylprednisolone in human blood samples were detected [33]. By contrast, according to [31], steroids, after four IT applications within a 10-12 day timeframe and a 2-3 days interval between each injection did not interfere with endogenous cortisol secretion or bone metabolism. We must note however that these papers concern short term therapy so long time effects are obscured. In our study no systemic adverse effects related to IT application of steroids were noticed. We experienced one case of an acute suppurative otitis media which was eliminated by use of local antibacterial therapy. All patients demonstrated a complete healing of MT after the tympanostomy tube removal.

Keeping in mind the options of ISSNHL steroid treatment and the benefitial and potential adverse effects, our experience confirms the crucial role of the need for a shared decision making situation of doctor with his patient.

\section{References}

1. Stachler JR, Chandrasekhar S, Archer S et al. Clinical practice guideline: sudden hearing loss. Otolaryngol Head Neck Surg. 2012 Mar; 146 (3 Suppl):1-35.

2. Powell-Tuck J, Bown RL, Lennard-Jones JE. A comparison of oral prednisolone given as single or multiple daily doses for active proctocolitis. Scand J Gastroenterol. 1978;13(7):833-837.

3. Rauch SD. Clinical practice: idiopathic sudden sensorineural hearing loss. N Engl J Med. 2008;359(8):833-840.

4. Parnes LS, Sun AH, Freeman DJ. Corticosteroid pharmacokinetics in the inner ear fluids: an animal study followed by clinical application. Laryngoscope. 1999;109(7, pt 2):1-17.

5. Chandrasekhar SS. Intratympanic dexamethasone for sudden sensorineural hearing loss: clinical and laboratory evaluation. Otol Neurotol 2001;22(1):18-23.

6. Rauch SD, Halpin CF, Antonelli PJ et al. Oral vs intratympanic corticosteroid therapy for idiopathic sudden sensorineural hearing loss: a randomized trial. JAMA. 2011 May 25;305(20):2071-9.

7. Plontke SK, Siedow N, Wegener R, Zenner HP, Salt AN. Cochlear pharmacokinetics with local inner ear drug delivery using a three-dimensional finite-element computer model. Audiol Neurotol 2007;12(1):37-48.

8. Plontke SK, Biegner T, Kammerer B, Delabar U, Salt AN. Dexamethasone concentration gradients along scala tympani after application to the round window membrane. Otol Neurotol 2008;29(3):401-6.

9. Plontke SK, Salt AN. Quantitative interpretation of corticosteroid pharmakokinetics in innner ear fluids using computer simulations. Hear Res 2003;182(1-2):34-42.

10. Plontke SK, Lцwenheim H, Mertens J et al. Randomized, double blind, placebo controlled trial on the safety and efficacy of continuous intratympanic dexamethasone delivered via a round window catheter for severe to profound sudden idiopathic sensorineural hearing loss after failure of systemic therapy. Laryngoscope 2009;119(2):359-69.

11. Salt AN. Dexamethasone concentration gradients along scala tympani after application to the round window membrane. Otol Neurotol 2008;29(3):401-6. 
12. Banerjee A, Parnes LS. Intratympanic corticosteroids for sudden idiopathic sensorineural hearing loss. Otol Neurotol 2005;26(5):878-81.

13. Gallant C, Kenny P. Oral glucocorticoids and their complications. A review. J Am Acad Dermatol 1986;14(2 Pt 1):161-77.

14. Salt AN, Plontke SK. Local inner-ear drug delivery and pharmakokinetiks. Drug Discov Today 2005;10(19):1299-306.

15. Byl FM. Sudden hearing loss: eight years' experience and suggested prognostic table. Laryngoscope 1984;94(5 Pt 1):647-61.

16. Battaglia A, Burchette R, Cueva R. Combination therapy (intratympanic dexamethasone + high-dose prednisone taper) for the treatment of idiopathic sudden sensorineural hearing loss. Otol Neuroto. 2008;29(4):453-60.

17. Loughan S. Management of sudden sensorioneural hearing loss: a consultant survey. J Laryngol Otol 2000;114(11):837-9.

18. Mattox DE, Simmons FB. Natural history of sudden sensorioneural hearing loss. Ann Otol Rhinol Laryngol 1977;86 (4 Pt 1):463-80.

19. Stokroos RJ, Albers FW, Schirm J. Therapy of idiopathic sudden sensorioneural hearing loss: antiviral treatment of experimental herpes simplex virus infection of the inner ear. Ann Otol Rhinol Laryngol 1999;108(5):423-8.

20. Zhao H, Zhang TY, Jing JH, Fu YY, Luo JN. Prognostic factors for patients with the idiopathic sudden sensorineural hearing loss [in Chineese]. Zhonghua Er Bi Yan Hou Tou Jing Wai Ke Za Zhi 2008;43(9):660-4.

21. Ho HG, Lin HC, Shu MT, Yang CC, Tsai HT. Effectiveness of intratympanic dexamethasone injection in sudden-deafness patients as salvage treatment. Laryngoscope 2004;114(7):1184-9.

22. Herr BD, Marzo SJ. Intratympanic steroid perfusion for refractory sudden sensorineural hearing loss. Otolaryngol Head Neck Surg 2005;132(4):527-31.

23. Choung YH, Park K, Shin YR, Cho MJ. Intratympanic dexamethazone injection for refractory sudden sensorineural hearing loss. Laryngoscope 2006;116(5):747-52.

24. Lefebvre PP, Staecker H. Steroid perfusion of the inner ear for sudden sensorineural hearing loss after failure of conventional therapy: a pilot study. Acta Otolaryngol 2002;122(7):698-702.

25. Plontke S, Lцwenheim H, Preyer S et al. Outcomes research analysis of continuous intratympanic glucocorticoid delivery in patients with acute severe to profound hearing loss: basis for planning randomized controlled trials. Acta Otolaryngol 2005;125(8):830-9.

26. Plaza G, Herrбiz C. Intratympanic steroids for treatment of sudden hearing loss after failure of intravenous therapy. Otolaryngol Head Neck Surg 2007;137(1):74-8.

27. Peng Y, Xiong S, Cheng Y, Qi YF, Yang Y. Clinical investigation of different routes of administration of dexamethasone on sudden deafness [in Chineese]. Lin Chung Er Bi Yan Hou Tou Jing Wai Ke Za Zhi 2008;22(10):442-5.

28. Kakehata S, Sasaki A, Oji K, et al. Comparison of intratympanic and intravenous dexamethasone treatment of sudden sensorineural hearing loss with diabetes. Otol Neurotol $2006 ; 27(5): 604-8$.

29. Alexander TH, Weisman MH, Derebery JM, et al. Safety of high-dose corticosteroids for the treatment of autoimmune inner ear disease. Otol Neurotol. 2009;30(4):443-448.

30. McDonough AK, Curtis JR, Saag KG. The epidemiology of glucocorticoid-associated adverse events. Curr Opin Rheumatol. 2008;20(2):131137.

31. Novoa E, Gaertner M, Henzen C. Systemic effects of the intratympanic dexamethasone therapy. Endocr Connect. 2014;1 3(3):127-131.

32. Chandrasekhar SS, Rubinstein RY, Kwartler JA et al. Dexamethasone pharmakineticsin the inner ear: comparison of the route of administration and use of facilitating agents. Otolaryngology-Head and Neck surgery. 2000;4:521-528.

33. Bird PA, Begg EJ, Zhang M, Keast AT, Murray DP, Balkany TJ. Intratympanic versus intravenous delivery of methylprednisolone to cochlear perilymph. Otology Neurootol. 2007:8:1124-1130.

\section{Corresponding author:}

prof. Sergey Kosyakov,

125057, 7 Ivan'kovskoe shosse, Moscow, Russia,

Russian Medical Academy of Postgraduate Education, ENT chair.

e-mail: serkosykov@yandex.ru,

mob. phone: +79166808883 . 